\title{
Isometric actions of Lie subgroups of the Moebius group
}

\author{
Charles Boubel and Abdelghani Zeghib \\ Unité de Mathématiques Pures et Appliquées (CNRS UMR 5669), École Normale Supérieure de \\ Lyon, 46 Allée d'Italie, F-69364 Lyon Cedex 07, France \\ E-mail: Charles.Boubel@ens-lyon.fr and Abdelghani.Zeghib@ens-lyon.fr
}

Received 28 October 2003, in final form 11 May 2004

Published 11 June 2004

Online at stacks.iop.org/Non/17/1677

doi:10.1088/0951-7715/17/5/006

Recommended by V Baladi

\begin{abstract}
We prove here, by geometric, or rather dynamical, methods, the following theorem. Let $G$ be a non-compact connected Lie subgroup of the isometry group Isom $\left(\mathbb{H}^{n}\right)$ of the real hyperbolic space $\mathbb{H}^{n}$, which does not fix any point at infinity, i.e. on $\partial \mathbb{H}^{n} \simeq \mathbb{S}^{n-1}$. Then $G$ preserves a certain hyperbolic subspace $\mathbb{H}^{d} \subset \mathbb{H}^{n}$ and 'contains' all the identity components $\operatorname{Isom}^{0}\left(\mathbb{H}^{d}\right)$ of its isometry group. We provide an 'algebra-free' proof and present the dynamical tools used, so that the exposition is 'self-contained'.
\end{abstract}

Mathematics Subject Classification: 53A30, 37C85, 57S20, 53C29

\section{Introduction}

We provide here a geometric_essentially dynamic_-proof of the following result.

Theorem 1.1. Let $G$ be a non-compact connected Lie subgroup of $\operatorname{Isom}\left(\mathbb{H}^{n}\right)$, which does not fix any point at infinity (i.e. on $\partial \mathbb{H}^{n} \simeq \mathbb{S}^{n-1}$ ). Then, up to conjugacy, G preserves a hyperbolic subspace $\mathbb{H}^{d}$ with $1 \leqslant d \leqslant n$ and contains $\mathrm{O}^{0}(d, 1)$.

In [DO], Di Scala and Olmos proved some equivalent results, describing additionally the case where $G$ admits a fixed point. Their motivation was, like ours, to give a geometrical proof of a result classically proved by algebraic means. This takes place in a more general background which we recall in section 1.1. However, some non-trivial algebraical tools are used in their proof. We propose here an 'algebra-free' approach (actually, we allow the use of some rudimentary notions, such as the radical of a Lie group, to show that theorems 1.1 and 1.2 are essentially equivalent). From a pedagogical view, the proof is fully self-contained (see section 1.2) and one of its steps is linked with a more general problem in the dynamics 
of algebraic groups (see section 1.3). Finally, we present an application of theorem 1.1 to the holonomy of Lorentzian manifolds (see section 1.4).

\subsection{Isometric dynamics on Riemannian symmetric spaces}

Let $X$ be a Riemannian symmetric space of non-positive curvature. The following two properties of subgroups of $\operatorname{Isom}(X)$ are well known. First, if $G$ is compact, then it has a fixed point in $X$. Second, if $G$ is a semi-simple (e.g. diagonalizable) one-parameter group, then it keeps invariant a geodesic, which is moreover unique in the negatively curved case and called the axis of $G$. The original proofs were algebraic in nature, but now there are also geometrical ones, applying to any Hadamard space [BGS].

However, both these facts are corollaries of the following less known statement.

Theorem 1.2. Let $G$ be a semi-simple Lie group acting isometrically on a Riemannian symmetric space $X$ of non-positive curvature. Then $G$ admits a totally geodesic orbit $Y$ (which is obviously a symmetric space). Moreover $G$ contains the identity component of the isometry group of $Y$; more precisely, the group of the restrictions of elements of $G$ to $Y$ contains the 'intrinsic' group $\operatorname{Isom}^{0}(Y)$.

Though this statement is purely geometric, only algebraic approaches to this result are found in the literature, with the exception of [DO]. One can find in [K] an algebraic proof of the first part of theorem 1.2 (the existence of a totally geodesic orbit); this reference is recalled in [AVS], theorem 3.7, p 103, where theorem 1.2 is stated in the case of constant curvature. We thank de la Harpe for this remark. Also, an algebraic proof of a proposition implying the second part of theorem 1.2, in the case $G=\mathrm{O}(n, 1)$, appears in [BH], section 3, proposition 1 .

The available formulations of theorem 1.2 are mostly algebraic. For example, for the 'universal' symmetric space $\operatorname{SL}(n, \mathbb{R}) / \mathrm{SO}(n)$, up to conjugacy, $G$ is adjoint, i.e. invariant under the canonical automorphism $A \mapsto\left(A^{*}\right)^{-1}$ of $\operatorname{SL}(n, R)$. In this case, the orbit of base point 1 is totally geodesic. Even this geometrical interpretation is not well known (see, for instance [GP], where the algebraic formulation is attributed to [Mo]).

Now, all proofs of the different algebraic formulations of theorem 1.2 use non-trivial results from the classification of semi-simple Lie groups, or at least significant steps towards it.

We would like to give a geometric proof of theorem 1.2. This would emphasize exactly which properties are behind it; also, as a by-product, one should get geometric proofs of other well-known algebraic facts regarding semi-simple Lie groups.

\subsection{Philosophy of this paper}

The essential mathematical aim of this paper is to realize the above wish in the particular case of hyperbolic space. More precisely, we prove the slightly different version, theorem 1.1, given at the beginning, which implies it (see lemma 2.1, section 2). We recognize this is modest with respect to the global goal. However:

- Paying attention to this question is by itself interesting. Beyond the technical content, it forces one to think about the 'geometrization of Lie groups'.

- In our exposition, we have tried to present the proof, based on geometric (essentially dynamic) arguments, in a completely self-contained way. For ease of reading, the background and proofs of the few standard lemmas used here are also recalled. The geometric (this time in a more analytical sense) motivation of [DO] was similar, yet this paper still uses (implicitly) non-trivial algebraic tools in the proofs, e.g. the Iwasawa decomposition. 
- Step two of the proof is an elementary result on conformal dynamics on the sphere, which suggests more serious questions in this special case as well as in the general case of boundaries of symmetric spaces (see section 1.3).

- We are confident that the hyperbolic part of the proof can be adapted to the general rank 1 case, and this would naturally involve beautiful geometric properties of the boundaries.

\subsection{Dynamics on boundaries, one step of the proof}

Step 2 of the proof rests on a rigidity property (lemma 4.1) of invariant sets under conformal dynamics on the sphere $\mathbb{S}^{n-1}$. Let us state it here-in a slightly different and weaker form-in order to comment on it. Denote by $\mathcal{M}_{d}^{k}$ the space of $C^{k}$ embedded compact submanifolds of dimension $d$ in $\mathbb{S}^{n-1}$ and by $\mathcal{S}_{d}$ the space of the $d$-dimensional (round) spheres.

Fact 1.3 (quick and weaker version of lemma 4.1). If $V \in \mathcal{M}_{d}^{1}$ is invariant under a noncompact subgroup of $\operatorname{Conf}\left(\mathbb{S}^{n-1}\right)$, then $V \in \mathcal{S}_{d}$.

Let us make the following observations.

(1) Other symmetric spaces. It is very useful to consider how this fact comes into play on other symmetric spaces.

Lemma 4.1, in step 2 of the proof, consists of finding an orbit of $G$ in $\mathbb{S}^{n-1}$, which is a (round) sphere. One then fills it (taking its convex hull) and gets a hyperbolic subspace. In the most general case (say for the symmetric space $\operatorname{SL}(n, \mathbb{R}) / \operatorname{SO}(n)$ ), algebraic geometric dynamics yields closed orbits of $G$ on the boundary. The filling process is then sufficiently regular in the general rank 1 case but delicate in higher rank. In any case, it seems not to be easy to describe the geometry of submanifolds of the boundary that are the boundary of geodesic submanifolds of the symmetric space. Even in the case of the complex hyperbolic space $\mathbb{H}_{\mathbb{C}}^{n}$, these boundary submanifolds may be quite complicated (see, for instance [G]).

(2) Stability. One may ask if a stable version of fact 1.3 holds. Indeed, it turns out that the following is true; we shall provide the details of its proof elsewhere.

Theorem 1.4. Endow $\mathcal{M}_{d}^{1}$ with the $C^{0}$ topology and consider the action of $\operatorname{Conf}\left(\mathbb{S}^{n-1}\right)$ on it. This is proper on $\mathcal{M}_{d}^{1} \backslash \mathcal{S}_{d}$ (and obviously transitive and non-proper on $\mathcal{S}_{d}$ ).

This means, roughly speaking, that there is a good complete system of conformal invariants for elements of $\mathcal{M}_{d}^{1} \backslash \mathcal{S}_{d}$.

(3) Topological submanifolds, fractals. The example of limit sets of quasi-Fuchsian groups shows that no rigidity condition extends to $\mathcal{M}_{d}^{0}$. It remains nevertheless interesting to understand the conformal dynamics on $\mathcal{M}_{d}^{0}$.

\subsection{An application: the holonomy of the Lorentz symmetric spaces}

Finally, let us mention an application of theorem 1.1, already indicated in [DO]. We state the result in terms of linear algebra.

Theorem 1.5. Let $G$ be a non-compact connected Lie subgroup of $\mathrm{O}(n, 1)=\mathrm{O}\left(\mathbb{R}^{n+1}, g\right)$, $g=-\mathrm{d} x_{0}^{2}+\mathrm{d} x_{1}^{2}+\cdots+\mathrm{d} x_{n}^{2}$, which does not preserve any isotropic direction. Then

- $G$ preserves a subspace $E \subset \mathbb{R}^{n+1}$, with $\operatorname{sgn}\left(g_{\mid E}\right)=(d, 1)$ and $\operatorname{dim} E=d+1 \geqslant 2$;

- $E$ and $G$ are such that $\left\{h_{\mid E} / h \in G\right\} \supset \operatorname{SO}^{0}\left(g_{\mid E}\right) \simeq \operatorname{SO}^{0}(d, 1)$. 
Corollary 1.6. Let $\mathrm{Hol}^{0}$ be the restricted holonomy group of an indecomposable (i.e. not locally decomposable into a Riemannian product) Lorentzian manifold of dimension $n$. Then either $\mathrm{Hol}^{0}=\mathrm{SO}^{0}(n-1,1)$ or $\mathrm{Hol}^{0}$ stabilizes an isotropic direction.

This fact was known since 1957, as a consequence of Berger's list [B2] of the possible holonomy groups for symmetric spaces. However, Bérard Bergery and Ikemakhen asked for a direct proof in [BBI], remark, p 31. As indicated in [BBI], Berger's theorem [B1] implies the above corollary for non-locally symmetric Lorentzian spaces. Now, if the manifold is locally symmetric and indecomposable, its isotropy subgroup Iso $^{0}$ is indecomposable, and so it can neither fix a non-isotropic vector (so cannot be compact) nor stabilize a subspace $E$ as in the statement of theorem 1.5. So it has to fix an isotropic direction. Now for locally symmetric spaces, $\mathrm{Hol}^{0} \subset \mathrm{Iso}^{0}$. The result follows. (Note also [Z] for another proof.)

\section{Background and structure of this paper}

\subsection{Some brief recalls}

Throughout, $g$ is the Lorentzian metric $-\mathrm{d} x_{0}^{2}+\mathrm{d} x_{1}^{2}+\cdots+\mathrm{d} x_{n}^{2}$ on $\mathbb{R}^{n+1}$ ( $d$ replacing $n$ in section 5). The $n$-dimensional hyperbolic space is $\mathbb{H}^{n}=\left\{m \in \mathbb{R}^{+} \times \mathbb{R}^{n} / g(m, m)=-1\right\}$, endowed with its Riemannian metric $g_{\text {hyp }}=g_{\mid \mathbb{H}^{n}}$, of constant curvature -1 . So $\operatorname{PO}(n, 1)$ is its isometry group Isom $\left(\mathbb{H}^{n}, g_{\text {hyp }}\right)$. Hence, $\mathrm{O}(n, 1)$ acts on $\mathbb{S}^{n-1}$, which bounds $\mathbb{H}^{n}$ at infinity. This action is conformal for the canonical conformal structure of $\mathbb{S}^{n-1}$; more precisely, $\operatorname{PO}(n, 1)$ is the conformal group Conf $\left(\mathbb{S}^{n-1}\right)$ of $\mathbb{S}^{n-1}$. Indeed, any sphere $\mathbb{S}^{n-1}$ imbedded into the $g$-isotropic cone of $\mathbb{R}^{n+1}$, transversally to the isotropic lines, inherits from $g$ a Riemannian metric. These inherited metrics are conformally equivalent to the canonical metric of $\mathbb{S}^{n-1}$. Finally, the topology on $\overline{\mathbb{H}}^{n}=\mathbb{H}^{n} \cup \mathbb{S}^{n-1}$ is the topology induced by that of $\mathbb{R}^{n}$ on the closed unit ball $\overline{\mathbb{D}}^{n}$, with which $\overline{\mathbb{H}}^{n}$ is identified by the stereographic projection of $\mathbb{R}^{n+1}$ on $\{0\} \times \mathbb{R}^{n}$, of pole $(-1,0, \ldots, 0)$. This topology gives naturally back, on $\mathbb{H}^{n}$ and $\mathbb{S}^{n-1}$, their canonical topology. Note that other topologies on $\overline{\mathbb{H}}^{n}$ with these properties exist and are used. An inversion centred at a point of the boundary of $\overline{\mathbb{D}}^{n}$ maps $\overline{\mathbb{D}}^{n}$ on the Poincaré half-space $\left(\simeq \mathbb{R}^{+} \times \mathbb{R}^{n-1}\right)$, another conformal model for $\mathbb{H}^{n}$.

The space of the oriented geodesics of $\mathbb{H}^{n}$ is parametrized by the couples of their limit points in $\mathbb{S}^{n-1}=\partial \mathbb{H}^{n}$, and so this space is identified with $\left(\mathbb{S}^{n-1}\right)^{2} \backslash \Delta$, where $\Delta$ is the diagonal, i.e. the graph of $\operatorname{Id}_{\mathbb{S}^{n-1}}$. The application $\Phi: T^{1} \mathbb{H}^{n} \rightarrow\left(\mathbb{S}^{n-1}\right)^{2} \backslash \Delta$ associating, to each vector $v \in \mathrm{T}^{1} \mathbb{H}^{n}$, the geodesic with initial condition $v$ is a $\mathcal{C}^{\infty}$ submersion; its fibres are naturally the orbits of the geodesic flow $\varphi^{t}$ of $\mathrm{T}^{1} \mathbb{H}^{n}$.

An element $h \in \mathrm{O}(n, 1)$ is said to be elliptic if it fixes a point of $\mathbb{H}^{n}$, i.e. if it admits an eigenvector $v \in \mathbb{R}^{n+1}$ such that $g(v, v)<0$. Else, it must fix a point of $\mathbb{S}^{n-1}$, i.e. admit a $g$-isotropic eigenvector $v$. Two cases are then possible: either this fixed point is unique (then $h(v)=v$ and $v \in \operatorname{Im}(h-\operatorname{Id}))$ and $h$ is said to be parabolic or $h$ admits exactly two fixed points, corresponding to two isotropic eigenvectors $v$ and $v^{\prime}$ in $\mathbb{R}^{n+1}$ with $h(v)=\lambda v$ and $h\left(v^{\prime}\right)=\lambda^{-1} v^{\prime}$ with $\lambda<1$; then $h$ is said to be loxodromic.

As stated in section 1.2, theorem 1.1 implies theorem 1.2. Actually, they are essentially equivalent. Let us recall briefly why (the following lemma holds in the general rank 1 case but not in a higher rank).

Lemma 2.1. If $G$ is a semi-simple non-compact connected subgroup of $\operatorname{Isom}\left(\mathbb{H}^{n}\right)$, it has no fixed point (neither at infinity nor in the interior).

If $G$ has no fixed point, then it fixes some hyperbolic subspace $\mathbb{H}^{d}$, and the so obtained homomorphism $G \rightarrow \operatorname{Isom}\left(\mathbb{H}^{d}\right)$ has a compact kernel and a semi-simple image. 
Proof. Assume $G$ fixes a point $x$. If $x \in \mathbb{H}^{n}, G$ is compact as it is contained in the stabilizer of $x$ that is isomorphic to $\mathrm{O}(n)$. If $x \in \partial \mathbb{H}^{n}$, its stabilizer is naturally isomorphic to the similarity group of a Euclidean space (see the model of the Poincaré half-space, with $x=\infty$ ) that cannot contain a non-compact semi-simple Lie group.

Conversely, let $R$ be the solvable radical of $G$. It will have a fixed point in $\overline{\mathbb{H}}=\mathbb{H} \cup \partial \mathbb{H}$. This is true in general and essentially equivalent to the fact that representations of solvable Lie groups preserve flags (see for instance [BGS] for an overview of the subject).

If $R$ is non-compact, then it contains some hyperbolic or parabolic element (see lemma 3.1 in section 3). The set of fixed points of such an element (parabolic or hyperbolic) consists of one or two points. Therefore, this property holds for the set of fixed points of $R$. The group $G$ acts on it and thus, by connectedness, fixes some point at infinity. This is a contradiction, and so $R$ must be compact. In this case, its fixed point set is a geodesic hyperbolic subspace $\mathbb{H}^{d}$. This determines a homomorphism $G \rightarrow \operatorname{Isom}\left(\mathbb{H}^{d}\right)$. Its kernel is compact since all its elements fix some interior point. Its image is semi-simple since this procedure kills the radical. By definition, the quotient of a Lie group by its radical has no radical and is therefore semi-simple.

Now the proof of theorem 1.5 is divided into one (classical) preliminary step and two additional steps, each of them corresponding to one section of this paper.

\section{Scheme of the proof.}

Step 1. Preliminary. We recall, with a sketch of its proof, a standard sufficient condition for the existence of loxodromic elements in a subgroup of $\mathrm{O}(n, 1)$.

Step 2. 'Rigidity' of some $G$-orbits on $\mathbb{S}^{n-1}$. We exhibit here a subspace $E$ as announced in the theorem. More exactly, we will prove that $G$ stabilizes a subsphere of $\mathbb{S}^{n-1}=\partial \mathbb{H}^{n}$, and hence its convex hull in $\mathbb{H}^{n}$, which is a hyperbolic subspace of $\mathbb{H}^{n}$, that is $H=\mathbb{H}^{n} \cap E$, where $E$ is a sub-vectorspace of $\mathbb{R}^{n+1}$. Moreover, $G$ acts transitively on this subsphere.

Step 3. 'Maximality' of $G$ in the stabilizer of $E$. We show how this leads to the result.

\section{Step 1 (standard): existence of loxodromic elements in $G$}

We prove the following lemma - the notion of the limit set is defined below.

Lemma 3.1. If $G$ is a non-precompact subgroup of $\mathrm{O}(n, 1), G$ has a loxodromic or a parabolic element. If its limit set, $L(G)$, contains two distinct points $x, y$ (so in particular if $G$ stabilizes no isotropic direction), $G$ contains a loxodromic transformation, the two fixed points of which are arbitrarily near $x$ and $y$.

This result is a consequence of a few classical (and basic) notions and results in hyperbolic dynamics. You can find a good introduction to them, for example, in [Ma], in the framework of flat conformal geometry, or in [EN], in that of Hadamard manifolds. The first notion is that of the limit set of $G$, which is most usually used for discrete groups but works in the same way for any group with the following definition.

Definition 3.2. The limit set, $L(G)$, of a group $G$ acting isometrically on $\mathbb{H}^{n}$ is $L(G)=$ $\mathbb{S}^{n-1} \cap \overline{G \cdot a}$, where $\mathbb{S}^{n-1}$ is the boundary of $\mathbb{H}^{n}$ at infinity and where $\overline{G \cdot a}$ is the closure, in $\overline{\mathbb{H}}^{n}$, of the $G$-orbit of a point $a \in \mathbb{H}^{n}$. This definition does not depend on the choice of a.

Definition 3.3. Two points $x$ and $y$ (not necessarily distinct) of $L(G)$ are called dual if there exists a sequence $\left(\varphi_{n}\right)_{n \in \mathbb{N}}$ of elements of $G$ such that, for one point $a \in \mathbb{H}^{n}$ (and then for any 
such point), $\varphi_{n}(a) \underset{n \rightarrow \infty}{\longrightarrow} x$ and $\varphi_{n}^{-1}(a) \underset{n \rightarrow \infty}{\longrightarrow} y$. We denote by $D(x)$ the set of the points of $\mathbb{S}^{n-1}$ dual to $x$.

We summarize the key facts needed in our proof in the following lemma. Its first point gives the 'independence of $a$ ' in the definitions.

\section{Lemma 3.4.}

(i) If $a, b \in \mathbb{H}^{n}, x \in \mathbb{S}^{n-1}=\partial \mathbb{H}^{n},\left(\varphi_{n}\right)_{n \in \mathbb{N}} \in \mathrm{O}(n, 1)^{\mathbb{N}}$ and $\varphi_{n}(a) \underset{n \rightarrow \infty}{\longrightarrow} x$, then $\varphi_{n}(b) \underset{n \rightarrow \infty}{\longrightarrow} x$.

(ii) For all $x$ in $\mathbb{S}^{n-1}, D(x)$ is G-invariant.

(iii) If $G$ fixes no point on $\mathbb{S}^{n-1}$, any two points of $L(G)$ are dual.

(iv) If $x, y$ in $\mathbb{S}^{n-1}$ are dual, $G$ has a parabolic or a loxodromic element. If $x \neq y$, G contains a loxodromic element $\varphi$, the two fixed points of which are arbitrarily near $x$ and $y$.

Proof. (i) This follows from the comparison between the hyperbolic and the Euclidean metrics on $\mathbb{D}^{n}:$ if $x \in \mathbb{S}^{n-1}, \mathbb{D}^{n} \ni a_{n} \rightarrow x, \mathbb{D}^{n} \ni b_{n} \rightarrow x$ and $d_{\text {hyp }}\left(a_{n}, b_{n}\right)$ is bounded, $d_{\text {eucl }}\left(a_{n}, b_{n}\right) \rightarrow 0$.

(ii) Let us take $y$ dual to $x, a \in \mathbb{H}^{n}$ and $\varphi \in G$. By the definition of duality, there is a sequence $\left(\varphi_{n}\right)_{n \in \mathbb{N}} \in G^{\mathbb{N}}$ such that $\varphi_{n}(a) \underset{n \rightarrow \infty}{\longrightarrow} x$ and $\varphi_{n}^{-1}(a) \underset{n \rightarrow \infty}{\longrightarrow} y$. Then naturally $\varphi\left(\varphi_{n}^{-1}(a)\right) \underset{n \rightarrow \infty}{\longrightarrow} \varphi(y)$ and by (i), $\varphi_{n}\left(\varphi^{-1}(a)\right) \underset{n \rightarrow \infty}{\longrightarrow} x$, that is to say, $\varphi(y)$ is dual to $x$.

(iii) Note that if $E \subset \mathbb{S}^{n-1}$ is $G$-invariant, so is its convex hull $C(E)$ in $\mathbb{H}^{n}$, i.e. the convex hull of the union of the geodesics joining the pairs of points of $E$. Therefore, for any $a \in C(E), G \cdot a \subset C(E)$, and so $\overline{G \cdot a} \cap \mathbb{S}^{n-1} \subset E$. By (i), it implies that $L(G) \subset E$ as soon as $C(E) \neq \emptyset$, i.e. as $\sharp E \geqslant 2$. Now if $x \in L(G)$, by the compactness of $\bar{H}^{n}, D(x) \neq \emptyset$; if moreover $G$ has no fixed point in $\mathbb{S}^{n-1}, D(x)$, which is $G$-invariant, contains at least two points. Applying the remark, we get $D(x) \supset L(G)$. This shows (iii).

(iv) Let us take $x$ and $y$ dual points in $\mathbb{S}^{n-1}$, represented by two isotropic vectors $e_{x}$ and $e_{y}$ of $\mathbb{R}^{n+1}$, and $\left(\varphi_{n}\right)_{n \in \mathbb{N}}$, a sequence in $G$ such that $\varphi_{n}(a) \underset{n \rightarrow \infty}{\longrightarrow} x$ and $\varphi_{n}^{-1}(a) \underset{n \rightarrow \infty}{\longrightarrow} y$. Then let us take $e$, a point in $\mathbb{H}^{n}$, i.e. a vector in $\mathbb{R}^{n+1}$ with $g(e, e)=-1$, and $K=G_{e} \simeq \mathrm{O}(n)$, its stabilizer; $K$ is a maximal compact subgroup of $\mathrm{O}(n, 1)$. Let $\varphi_{n}=K_{n} A_{n} K_{n}^{\prime}$ be the decomposition of each $\varphi_{n}$ with $K_{n}$ and $K_{n}^{\prime}$ in $K$ and $A_{n} \in \mathrm{O}(n, 1)$ diagonal; note that $A$ has then at most two eigenvalues in $\mathbb{R} \backslash\{-1,1\}, \lambda_{n}$ and $\lambda_{n}^{-1}$, of multiplicity 1 . By the compactness of $K$, taking possibly a subsequence of $\left(\varphi_{n}\right)_{n \in \mathbb{N}}, K_{n}$ and $K_{n}^{\prime}$ tend, respectively, to some $K_{\infty}$ and $K_{\infty}^{\prime}$ in $K$. Now, as for $a \in \mathbb{H}^{n}, \varphi_{n}(a) \underset{n \rightarrow \infty}{\longrightarrow} x, A_{n}$ leaves all compact sets of $\mathrm{O}(n, 1)$, i.e. its eigenvalues $\lambda_{n}>1$ and $\lambda_{n}^{-1}$, associated to (isotropic) eigenvectors $e_{n}$ and $e_{n}^{\prime}$, leave all compact sets of $\mathbb{R}^{*}$. Let us denote by $x_{n}$ and $y_{n}$ the points of $\partial \mathbb{H}^{n}$ associated to $e_{n}$ and $e_{n}^{\prime}$. Then, for $n$ big enough, $\varphi_{n}\left(K_{\infty}^{\prime-1}(a)\right)$ must be close to $K_{\infty} x_{n}$. As it is close to $x$, necessarily $x_{n} \underset{n \rightarrow \infty}{\longrightarrow} K_{\infty}^{-1} x$. Similarly, $y_{n} \underset{n \rightarrow \infty}{\longrightarrow} K_{\infty}^{\prime} y$. Besides, if $\varepsilon>0$, if $B_{\varepsilon}^{\prime}$ and $B_{\varepsilon}$ are the Euclidean $\varepsilon$-balls of the respective centres $\mathbb{K}_{n}^{\prime-1} y_{n}$ and $\mathbb{K}_{n} x_{n}$ (in the model of the Poincaré ball $\mathbb{D}^{n}$ ), and if $\left|\lambda_{n}\right|$ is big enough, then $K_{n} A_{n} K_{n}^{\prime}$ sends $\overline{\mathbb{D}}^{n} \backslash\left(B_{\varepsilon}^{\prime} \cap \overline{\mathbb{D}}^{n}\right)$ inside $\overline{\mathbb{D}}^{n} \cap \bar{B}_{\varepsilon}$ ('north-south dynamics'). One checks then that $\varphi_{n}$ is parabolic or loxodromic with fixed point(s) $\varepsilon$-close to $K_{n}^{\prime-1} y_{n}$ and $K_{n} x_{n}$. If $x \neq y$, for $n$ big enough, these fixed point(s) are distinct, and so $\varphi_{n}$ is loxodromic.

Notes. The ' $K A K$ decomposition' - given by the existence of a square root of any symmetric matrix - shortens the proof of (iv). Alternate tools may be used, e.g. the notion of the 'vision angle' in a Hadamard manifold ([EN] propositions 8.4-8.6, 4.7 and 6.4) or of an isometric sphere of a conformal map ([Ma], section 5, notably lemma 5.8). 
Note that in general $G$ does not contain a parabolic or loxodromic element, the set of fixed points of which is $\{x, y\}$, even if $G$ is closed in $\mathrm{O}(n, 1)$. For example, if $G$ is a cocompact lattice, all pairs $\{x, y\} \subset \partial \mathbb{H}$ are dual, though only a countable number of them are the set of fixed points of elements of $G$.

Proof of lemma 3.1. If $L(G) \neq \emptyset$, the result follows from (iii) and (iv). Now $L(G)=\emptyset$ if and only if $G$ is precompact. The 'if' part is immediate; let us check the 'only if'. If $L(G)=\emptyset$, by the definition of $L(G)$, the orbit $G \cdot a$ of any $a \in \mathbb{H}^{n}$ is bounded. So is $\bar{G} \cdot a$, where $\bar{G}$ is the closure of $G$ in $\mathrm{O}(n, 1)$. Now, denoting by $\bar{G}_{a}$ the stabilizer of $a$ in $\bar{G}, \pi: \bar{G} \rightarrow \bar{G} / \bar{G}_{a}$ is a fibration over $\bar{G} / \bar{G}_{a}$, homeomorphic to $\bar{G} \cdot a$, and hence compact, with fibre $\bar{G}_{a}$, which is also compact, as included in $\mathrm{O}\left(\mathrm{T}_{a} \mathbb{H}^{n},\left(g_{\text {hyp }}\right)_{\mid a}\right) \simeq \mathrm{O}(n)$. So $\bar{G}$ is compact.

\section{Step 2: a rigidity result about differentiable submanifolds of $\mathbb{S}^{n-1}$}

The fundamental lemma on which the second step is based is the following.

Lemma 4.1. Let $\mathcal{M}$ be a connected differentiable manifold of dimension $k$ and $F: \mathcal{M} \rightarrow \mathbb{S}^{m}$ an injective continuous map of $\mathcal{M}$ into $\mathbb{S}^{m}$. Let us suppose that $\mathcal{F}=F(\mathcal{M})$ is $h$-invariant, where $h$ is a loxodromic transformation of $\mathbb{S}^{m}$ with attractive point $q_{+} \in \mathcal{F}$ and that $F$ is differentiable at $F^{-1}\left(q_{+}\right)$. Then $\mathcal{F}$ is a $k$-sphere of $\mathbb{S}^{m}$ passing by the poles $q_{+}$and $q_{-}$of $h$, or such a sphere, without the point $q_{-}$.

Proof of the lemma. A stereographic map from $\mathbb{S}^{m} \backslash\left\{q_{-}\right\}$onto $\mathbb{R}^{m}$ sending $q_{+}$on 0 conjugates $h$ with a linear transformation of $\mathbb{R}^{n}$ of the type $\lambda A$, where $\left.\lambda \in\right] 0,1[$ and $A \in \mathrm{O}(\mathrm{m})$. We go further in this framework.

Let us denote by $p$ the inverse image of $q_{+}$by $F$. Using the differentiability hypothesis about $F$, we may choose an open set $\mathcal{U}$ of $\mathcal{M}$ containing $p$ and such that, in a well-chosen coordinate system of $\mathbb{R}^{m}$, centred at $0, F(\mathcal{U})$ is the graph of a certain function $f: \mathbb{R}^{k} \rightarrow \mathbb{R}^{n-k}$, differentiable at 0 (and, for convenience, satisfying $f(0)=0$ ):

$$
F(\mathcal{U})=\left\{(x, y) \in \mathbb{R}^{k} \times \mathbb{R}^{m-k} / x \in B(0, r) \text { and } y=f(x)\right\} .
$$

Now, necessarily, $f$ is linear. Let us prove it.

Remark. The particular case $A=\mathrm{Id}$. In this case, the $h$-invariance of $\mathcal{F}$ implies that $f$ commutes with $x \mapsto \lambda x$. Now, lots of such functions exist, but only the linear ones may be differentiable at 0 . Indeed, by the definition of differentiability,

$$
\lambda^{-n} f\left(\lambda^{n} x\right) \underset{n \rightarrow \infty}{\longrightarrow} \mathrm{d} f(0) \cdot x
$$

and so, if $\lambda^{-n} f\left(\lambda^{n} x\right)=f(x), f$ has to be equal to its differential, $\mathrm{d} f(0)$.

The proof of the general case, modulo technical modifications, is the same as $\mathrm{O}(\mathrm{m})$ is compact. For convenience, let us choose more precise coordinates. The first factor, $\mathbb{R}^{k} \times\{0\}$, may be chosen equal to $\mathrm{T}_{q_{+}} \mathcal{F}(\mathcal{U})$, so that $\mathrm{d} f\left(q_{+}\right)=0$. Besides, as $\mathcal{F}=F(\mathcal{M})$ is $h$-stable, $\mathrm{T}_{q_{+}} \mathcal{F}(\mathcal{U})$ is $A$-stable. Choosing $\left(\mathrm{T}_{q_{+}} \mathcal{F}(\mathcal{U})\right)^{\perp}$ as second factor $\{0\} \times \mathbb{R}^{m-k}$, that factor is also $A$-stable, $A$ being orthogonal. Then

$$
\begin{aligned}
h(F(\mathcal{U}))= & \left\{A \cdot(x, y) \in \mathbb{R}^{k} \times \mathbb{R}^{m-k} / x \in B(0, \lambda r) \text { and } y=\lambda \cdot f\left(\lambda^{-1} \cdot x\right)\right\} \\
= & \left\{(x, y) \in \mathbb{R}^{k} \times \mathbb{R}^{m-k} / x \in B(0, \lambda r) \text { and } y=A^{\prime \prime} \lambda \cdot f\left(\lambda^{-1} \cdot A^{\prime-1} x\right)\right\}, \\
& \text { where } A^{\prime}=A_{\mid \mathbb{R}^{k} \times\{0\}} \text { and } A^{\prime \prime}=A_{\mid\{0\} \times \mathbb{R}^{m-k}} .
\end{aligned}
$$


Now, as $\mathcal{F}=F(\mathcal{M})$ is preserved by $h, h(F(\mathcal{U})) \subset \mathcal{F}$. As the projection from $\mathbb{R}^{m}$ on its first factor, $\mathbb{R}^{k}$, maps $h(F(\mathcal{U}))$ onto $B(0, \lambda r)$, necessarily

$$
h(F(\mathcal{U}))=\left\{(x, y) \in \mathbb{R}^{k} \times \mathbb{R}^{m-k} / x \in B(0, \lambda r) \text { and } y=f(x)\right\} .
$$

Therefore, on $B(0, r), f \circ \lambda A^{\prime}=\lambda A^{\prime \prime} \circ f$. Fixing an $x$ near 0 , we get, for any $n \in \mathbb{N}$,

$f\left(\lambda^{n} A^{\prime n} \cdot x\right)=\lambda^{n} A^{\prime \prime n} \cdot f(x), \quad$ or equivalently $\lambda^{-n} A^{\prime \prime-n} \cdot f\left(\lambda^{n} A^{\prime n} \cdot x\right)=f(x)$.

Now $f(0)=0, f$ is differentiable at 0 , the coordinates are such that $\mathrm{d} f(0)=0$, and $A^{\prime}$ and $A^{\prime \prime}$ preserve the Euclidean norm, and so $\lambda^{-n} A^{\prime \prime-n} \cdot f\left(\lambda^{n} A^{\prime n} \cdot x\right) \underset{n \rightarrow \infty}{\longrightarrow} 0$. Thus $f=0$ on $B(0, r)$; that is to say, $f$ is linear on $B(0, r)$, and hence $f \circ A^{\prime}=A^{\prime \prime} \circ f$.

Finally, as $\mathcal{F}$ is $h$-invariant, and thus $h^{-1}$ is also invariant, it contains $\mathcal{S}^{\prime}=$ $\bigcup_{n \in \mathbb{N}} h^{-n}(F(\mathcal{U}))$, which is $f\left(\mathbb{R}^{k}\right)$, a vectorspace of dimension $k$. Going back to $\mathbb{S}^{m}$ by the inverse of the stereographic map, $\mathcal{S}^{\prime}=\mathcal{S} \backslash\left\{q_{-}\right\}$, where $\mathcal{S}$ is a $k$-sphere passing by $q_{+}$and $q_{-}$. As the only proper extension, as an immersed manifold, of $\mathcal{S}^{\prime}$ in $\mathbb{S}^{m}$, is $\mathcal{S}$, and as $\mathcal{M}$ is connected, $\mathcal{F}=\mathcal{S}$ or $\mathcal{F}=\mathcal{S}^{\prime}=\mathcal{S} \backslash\left\{q_{-}\right\}$.

Proof of step 2. The group $G$ is supposed to preserve no isotropic direction, that is to say it has no fixed point on $\mathbb{S}^{n-1}=\partial \mathbb{H}^{n}$. So by lemma 3.1, there is a loxodromic element $\gamma$ in $G$. Let $q_{+} \in \mathbb{S}^{n-1}$ be the attractive point of $\gamma$ and $G_{q_{+}} \subset G$ the stabilizer of $q_{+}$in $G$. We apply lemma 4.1 to the $G$-orbit of $q_{+}$, i.e. with $\mathcal{M}=G / G_{q_{+}}$and $F: h \mapsto h\left(q_{+}\right)$, and so $\mathcal{F}=F(\mathcal{M})$ is the $G$-orbit $G \cdot q_{+}$of $q_{+}$. So $G \cdot q_{+}$is a certain sphere $\mathcal{S}$ or $\mathcal{S} \backslash\left\{q_{-}\right\}$. The second case is impossible, for $\left\{q_{-}\right\}=$bound $\left(\mathcal{S} \backslash\left\{q_{-}\right\}\right)$would be $G$-invariant. So $G \cdot q_{+}$is a $G$-invariant sphere of $\mathbb{S}^{n-1}$, and step 2 is achieved.

\section{Step 3: 'maximality' of $G$}

We prove now the end of the statement of theorem 1.5, which is as follows.

Proposition 5.1. Let $G$ be a non-compact (closed) connected Lie subgroup of $\operatorname{SO}^{0}(d, 1)$ that acts transitively on $\mathbb{S}^{d-1}$. Then $G=\operatorname{SO}^{0}(d, 1)$.

By lemma 3.1, section 3, we can take a loxodromic element $\gamma$ in $G$. The space of the geodesics of $\mathbb{H}^{d}$ is diffeomorphic to $\left(\mathbb{S}^{d-1}\right)^{2} \backslash \Delta$; with this identification, the axis, $\theta$, of this loxodromic element, $\gamma$, corresponds to the couple $\left(q_{-}, q_{+}\right)$of its endpoints. Step 3 rests on two lemmas.

Lemma 5.2. The G-orbit of $\theta$ is open in the space of the geodesics of $\mathbb{H}^{d}$.

Proof. Let $\pi_{1}$ and $\pi_{2}$ be the restrictions to $\left(\mathbb{S}^{d-1}\right)^{2} \backslash \Delta$ of the projections on the first, respectively, second, factor $\mathbb{S}^{d-1}$. Let us also set $q=\left(q_{-}, q_{+}\right)$. After Step $2, G \cdot q_{+}=\mathbb{S}^{d-1}$, and so $\pi_{2}(G \cdot q)=G \cdot q_{+}=\mathbb{S}^{d-1}$ and symmetrically $\pi_{1}(G \cdot q)=\mathbb{S}^{d-1}$.

Now $G \cdot q$ is the image of an immersion of $G / G_{q}$ in $\left(\mathbb{S}^{d-1}\right)^{2} \backslash \Delta$, and so, differentiating this immersion at the identity, we get a subspace $F=\mathrm{T}_{q}(G \cdot q) \subset \mathrm{T}_{q}\left(\left(\mathbb{S}^{d-1}\right)^{2} \backslash \Delta\right)$, which is stable under the action of the stabilizer $G_{q}$. In particular, it is $\mathrm{d} \gamma(q)=\left(\mathrm{d} \gamma\left(q_{-}\right), \mathrm{d} \gamma\left(q_{+}\right)\right)$-stable, where $\gamma$ is seen, successively, as a diffeomorphism of $\left(\mathbb{S}^{d-1}\right)^{2}$ and of $\mathbb{S}^{d-1}$.

In a chart $(s, i \circ s)$ of $\left(\mathbb{S}^{d-1}\right)^{2}$, where $s$ is a stereographic map from $\mathbb{S}^{d-1}$ onto $\mathbb{R}^{d-1} \cup\{\infty\}$ sending $q_{-}$on 0 and $q_{+}$on $\infty$ and where $i$ is the inversion of $\mathbb{R}^{d-1}$ of centre 0 and radius 1 
(the radius does not matter), $\mathrm{d} \gamma(q)$ is represented by a linear isomorphism, $B$, of $\left(\mathbb{R}^{d+1}\right)^{2}$ of the type

$$
\left.B=\left(\begin{array}{cc}
\lambda^{-1} A & 0 \\
0 & \lambda A
\end{array}\right), \quad \text { where } A \in \mathrm{O}(d-1) \quad \text { and } \quad \lambda \in\right] 0,1[\text {. }
$$

The image, $E$, of $\mathrm{T}_{q}(G \cdot q)$ by this chart is $B$-invariant. Let us denote by $E_{1}$ and $E_{2}$ the first and second factors of the product $\left(\mathbb{R}^{d-1}\right)^{2}$ and by $\mathrm{pr}_{i}: E_{1} \oplus E_{2} \rightarrow E_{i}$ the canonical projections. By assumption, $\pi_{i}(G \cdot q)=\mathbb{S}^{d-1}$; differentiating, in the chart, these equalities in 0 , we get $\operatorname{pr}_{i}(E)=E_{i}$ for each $i$.

Remark. The particular case $A=\mathrm{Id}$. As $E$ is $B$-stable, it has immediately to be, in this case, $\{0\}, E_{1}, E_{2}$ or $E_{1} \oplus E_{2}$. As $\operatorname{pr}_{i}(E)=E_{i}$ for $i=1,2, E=E_{1} \oplus E_{2}=\left(\mathbb{R}^{d-1}\right)^{2}$, i.e. $\operatorname{dim} G \cdot q=2(d-1)$, which is the wanted result.

The general case works similarly. Since each $E_{i}$ is a sum of spectral subspaces of $B$ (associated with complex eigenvalues of the respective type $\lambda^{-1} \mathrm{e}^{\mathrm{i} \theta}$ and $\lambda \mathrm{e}^{\mathrm{i} \theta}, \theta \in \mathbb{R}$ ), the

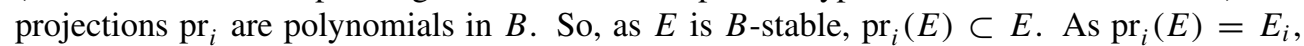
$E \supset E_{1} \oplus E_{2}$ and the result is arrived at as above.

Lemma 5.3. Take $G$ as in proposition 5.1, with $d \geqslant 2$. Then $G$ acts transitively on the unit tangent bundle $\mathrm{T}^{1} \mathbb{H}^{d}$ of $\mathbb{H}^{d}$.

Remark. In the case $d=1$, which we shall not need, the following proves the transitivity of $G$ on $\mathbb{H}^{d}$.

Proof. Throughout, we take $\gamma$, a loxodromic element of $G, \theta$, its axis, and $v$, a unit tangent vector to $\theta$, at some point $x$ of $\theta$.

As $G$ acts isometrically on $\mathbb{H}^{d}$, its action on $\mathrm{T}^{1} \mathbb{H}^{d}$ commutes with the geodesic flow $\varphi^{t}: \forall \gamma \in G, \forall t \in \mathbb{R}, \forall v \in \mathrm{T}^{1} \mathbb{H}^{d} \varphi^{t}(\gamma(v))=\gamma\left(\varphi^{t}(v)\right)$. In particular, $\Phi$ is $G$-equivariant, with $\Phi$ the canonical submersion from $\mathrm{T}^{1} \mathbb{H}^{d}$ onto $\left(\mathbb{S}^{d-1}\right)^{2} \backslash \Delta$, as set in the introduction. By lemma $5.2, \Phi(G \cdot v)=G \cdot \theta$ is open, and so

- either $G \cdot v$ is open in $\mathrm{T}^{1} \mathbb{H}^{d}$

- or $G \cdot v$ is transverse to the fibres of $\Phi$, that is to the orbits of the geodesic flow $\varphi^{t}$.

Now, as $\operatorname{Isom}\left(\mathbb{H}^{d}, g_{\text {hyp }}\right)$ acts properly on $\left(\mathbb{H}^{d}, g_{\text {hyp }}\right)$, it acts properly on its unit tangent bundle $\mathrm{T}^{1} \mathbb{H}^{d}$, and hence so does $G$. Consequently, the orbits of $G$ in $\mathrm{T}^{1} \mathbb{H}^{d}$ are closed.

So in the first case, $G \cdot v$ is open and closed in $\mathrm{T}^{1} \mathbb{H}^{d}$, which is connected, and so $G \cdot v=\mathrm{T}^{1} \mathbb{H}^{d}$ and we are done.

We complete the proof by supposing that the second case holds and by obtaining a contradiction. Before doing this, let us recall a classical fact about the loxodromic elements of $\operatorname{Isom}\left(\mathbb{H}^{d}, g_{\text {hyp }}\right)$.

Recall. If $h$ is a loxodromic element of Isom $\left(\mathbb{H}^{d}, g_{\text {hyp }}\right)$ and $\theta_{h}$ its axis, then $h_{\mid \theta_{h}}$ is a translation. The (constant) distance $\tau(h)=d(h(m), m)$, for $m \in \theta_{h}$, is equal to $2 \ln (\rho(h))$, where $\rho(h)>1$ is the spectral radius of $h \in \mathrm{O}(d, 1)$.

As $G$ and $\varphi^{t}$ commute, we may associate to each vector $w$ of $\mathrm{T}^{1} \mathbb{H}^{d}$ the group $T(w)=\left\{t \in \mathbb{R} ; \varphi^{t}(G \cdot w)=G \cdot w\right\}$. Let us consider $T(v)$. It is a subgroup of $(\mathbb{R},+)$, and so $T(v)=\{0\}, T(v)=a \mathbb{Z}$ for some $a \in \mathbb{R}$ or $T(v)$ is dense. The last case is impossible as $G \cdot v$ is transverse to $\varphi^{t}$ and closed. So is the first one: $\gamma(v)$ is still tangent, with the same orientation 
as $v$, to the axis, $\theta$, of $\gamma$ and $\gamma(v) \neq v$, and so there is a $t \in \mathbb{R}^{*}$ such that $\gamma(v)=\varphi^{t}(v)$ (actually $t=\tau(\gamma)$, see recall). So $\varphi^{\tau(\gamma)}(G \cdot v)=G \cdot v$ and $T(v) \supset \tau(\gamma) \mathbb{Z} \supsetneq\{0\}$.

Let us finally rule out the second case. As $G$ and $\varphi^{t}$ commute, $T$ is invariant along the orbits of $\varphi^{t}$ (CB thanks Thierry Barbot for this remark), and so $T$ may be viewed as a function of the (oriented or not: we choose 'oriented') geodesics of $\mathbb{H}^{d}$. For $\theta$ such a geodesic, let us set $a(\theta)=\inf \left(T(\theta) \cap \mathbb{R}_{+}^{*}\right)$. Trivially, $T$, and hence $a$, is invariant along the orbit $G \cdot \theta$ of $\theta$. If $h$ is a loxodromic element of $G$, of axis $\theta_{h}$, the same argument as used above for $v$ gives $a\left(\theta_{h}\right) \leqslant \tau(h)$.

By lemma 5.2, the $G$-orbits of such geodesics $\theta_{h}$ are open in $\left(\mathbb{S}^{d-1}\right)^{2} \backslash \Delta$, and so $T$ and hence $a$ are locally constant on the union, $\mathcal{U}$, of these orbits-note that $\mathcal{U}$ is simply the set of the (couples of endpoints of the) axis of loxodromic elements of $G$. Besides, an element $h$ of $\mathrm{O}(d, 1)$ is loxodromic if and only if $\rho(h)>1$, and so $\mathcal{U}=\rho^{-1}(] 1,+\infty[)$. As $G$ is connected and as Id $\in \rho^{-1}(1) \neq \emptyset$, the closure of each connected component of $\mathcal{U}$ contains $\rho^{-1}(1)$; in particular, if $\mathcal{U}_{1}$ is such a component, $\inf _{\mathcal{U}_{1}} \rho=1$. Let $a_{1}$ be the constant value of $a$ on $\mathcal{U}_{1}$; if $h \in \mathcal{U}_{1}$ and if $\theta_{h}$ is its axis, $a_{1}=a\left(\theta_{h}\right)<\tau(h)=2 \ln (\rho(h))$, and so $a_{1} \leqslant \inf _{\mathcal{U}_{1}}(2 \ln \circ \rho)=0$ : therefore $a=0$ on $\mathcal{U}$, which is a contradiction.

We will also need a little standard lemma, for which we give a proof.

Lemma 5.4. Let $G$ be a Lie group acting differentiably and transitively on a connected manifold $\mathcal{M}$. Then the identity component, $G^{0}$, of $G$ also acts transitively on $\mathcal{M}$.

Proof. Let $a$ be a point in $\mathcal{M}$. As $G$ is a Lie group, the $G^{0}$-orbit of $a$ is open in $G \cdot a=\mathcal{M}$, as well as the orbit of $a$ under the action of any connected component of $G$. As these orbits are pairwise disjoint or equal, they are also closed; in particular, $G^{0} \cdot a$ is closed. As $\mathcal{M}$ is connected, $G^{0} \cdot a=\mathcal{M}$.

Proof of proposition 5.1. By lemma 5.3, $G$ acts transitively on $T^{1} \mathbb{H}^{d}$. By induction, we will prove that $G \cap \mathrm{Isom}_{+}\left(\mathbb{H}^{d}, g_{\text {hyp }}\right)$ acts transitively on the fibre bundle $\mathcal{B}^{+} \mathbb{H}^{d}$ of the positive orthonormal frames on $\mathbb{H}^{d}$. As an isometry of $\left(\mathbb{H}^{d}, g_{\text {hyp }}\right)$ is determined by its differential at any point, it means that $G \supset \operatorname{Isom}_{+}\left(\mathbb{H}^{d}, g_{\text {hyp }}\right)=\operatorname{SO}^{0}(d, 1)$, which is the required result.

Let us take $v$, any element of $\mathrm{T}^{1} \mathbb{H}^{d}$, and $H_{1}=\exp \left(v^{\perp}\right)$, the hyperbolic hyperplane of $\mathbb{H}^{d}$, passing through the base point $x$ of $v$ and orthogonal to $v$. Let $G_{1} \subset G$ be the stabilizer of $H_{1}$ in $G$ and $G_{1}^{0}$ its identity component. Then $G_{1}^{0}$, which is a closed subgroup of $\operatorname{Isom}\left(H_{1}\right)$, as the condition $g\left(H_{1}\right) \subset H_{1}$ is closed, acts transitively on $H_{1}$. Let us check it.

Let us take $y \in H_{1}$ and $w$ one of the two unit normal vectors to $H_{1}$ at $y$. As $G$ acts transitively on $\mathrm{T}^{1} \mathbb{H}^{d}$, there is an $h$ in $G$ such that $h(v)=w$. The differential, $\mathrm{d} h$, of $h$ maps $v^{\perp}$ on $w^{\perp}$, and so $h$ maps $H_{1}=\exp \left(v^{\perp}\right)$ on $H_{1}=\exp \left(w^{\perp}\right)$ - both are $H_{1}$ as $H_{1}$ is totally geodesic-i.e. $h \in G_{1}$; $h$ maps the base point, $x$, of $v$ on $y$, and so $G_{1}$ acts transitively on $H_{1}$. So does $G_{1}^{0}$, by lemma 5.4. Note that $G_{1}^{0}$ cannot then be compact as $H_{1}$ is not compact; so it has loxodromic elements. We will moreover prove the following claim.

Claim. $G_{1}^{0}$ has no fixed point at infinity on $\partial H_{1} \simeq \mathbb{S}^{d-2}$.

Let us suppose the contrary: then this fixed point $p_{1}$ is unique; indeed else $G_{1}^{0}$ would stabilize any geodesic linking two such fixed points, which is excluded as $G_{1}^{0}$ is transitive on $H_{1}$. Thus, there is an application, $\psi$, associating, to each 'hyperbolic hyperplane' $H_{1}^{\prime}$ of $\mathbb{H}^{d}$, the fixed point of the identity component, $G_{H_{1}^{\prime}}^{0}$, of its stabilizer.

Recall. The set of the hyperbolic hyperplanes of $\mathbb{H}^{d}$ is parametrized by the set $\mathcal{E} \subset \mathbb{R} \mathrm{P}^{d}$ of the vectorial lines of $\mathbb{R}^{d+1}$, on which $g$ is positive definite. Indeed, $H_{1}^{\prime}$ is such a hyperplane if and 
only if it is of the form $\mathbb{H}^{d} \cap E^{\prime}$, where $E^{\prime}$ is a hyperplane of $\mathbb{R}^{d+1} \operatorname{such}$ that $\operatorname{sgn}\left(g_{\mid E^{\prime}}\right)=(d-1,1)$, i.e. such that $E^{\prime \perp} \in \mathcal{E}$. (Hence, $\mathcal{E}$ is canonically diffeomorphic to the de Sitter space $\mathrm{dS}^{d}=\left\{m \in \mathbb{R}^{d+1} ; g(m, m)=1\right\}$, quotiented by antipody $m \sim-m$ : a $\delta \in \mathcal{E}$ cuts $\mathrm{d} \mathrm{S}^{d}$ in two antipodal points.)

Now, $G$ is transitive on $\mathrm{T}^{1} \mathbb{H}^{d}$ and consequently also on the set $\left\{\exp \left(w^{\perp}\right) ; w \in \mathrm{T}^{1} \mathbb{H}^{d}\right\}$ of the hyperbolic hyperplanes of $\mathbb{H}^{d}$. So $\psi$, which is $G$-equivariant, is defined by $\psi\left(g \cdot H_{1}\right)=g \cdot p_{1}$, and so $\psi$ is differentiable and thus continuous.

Let us fix a geodesic $\theta$ of $H_{1}$, with endpoints $\left\{q_{-}, q_{+}\right\} \not \ngtr p_{1}$. If $\theta$ is the axis of some loxodromic element $\gamma$ of $G_{1}$, we are done. Indeed if so, let us take a loxodromic element $h$ in $G_{1}^{0}$ and denote its axis $\theta_{h} ; p_{1}$ is necessarily one of its endpoints. Then $\gamma h \gamma^{-1}$ is loxodromic with axis $\gamma\left(\theta_{h}\right): p_{1} \notin\left\{q_{-}, q_{+}\right\}, \gamma\left(p_{1}\right) \neq p_{1}$, and so $p_{1}$ is not an endpoint of $\gamma\left(\theta_{h}\right)$. So $\gamma h \gamma^{-1}\left(p_{1}\right) \neq p_{1}$, which cannot hold: as $G_{1}^{0}$ is normal in $G^{1}, \gamma h \gamma^{-1} \in G_{1}^{0}$. So we are left with finding a loxodromic element in $G_{1}$ with axis $\theta$.

As $G$ acts transitively on $\mathrm{T}^{1} \mathbb{H}^{d}$, the stabilizer, $G_{\theta}$, of $\theta$ in $G$ acts transitively on $\theta$; so does its identity component $G_{\theta}^{0}$, by lemma 5.4. Now, $\mathrm{O}(d, 1)_{\theta}^{0}$, the full connected stabilizer of $\theta$ in $\mathrm{O}(d, 1)$, is isomorphic to $\mathbb{R} \times \mathrm{SO}^{0}(d-1)$, with the first factor, $\mathbb{R}$, standing for the translation along $\theta$ (see, in the model of the Poincare half-space, the stabilizer of the half line $i \mathbb{R}_{+}^{*}$; we denote it additively, and so $(\mathbb{R},+))$. Note that an $h \in \mathrm{O}(d, 1)_{\theta}^{0} \simeq \mathbb{R} \times \mathrm{SO}^{0}(d-1)$ is loxodromic if and only if its component on $\mathbb{R}$ is not 0 . Now, $G_{\theta}^{0}$ is transitive on $\theta$, which exactly means that its projection on this first factor, $\mathbb{R}$, is surjective. So one can find a one-parameter subgroup $\Gamma=\left\{\gamma_{t} ; t \in \mathbb{R}\right\}$ of $G_{\theta}^{0}$ consisting, except Id, of loxodromic elements, and transitive on $\theta$.

Now let us choose an $x$ in $\theta$ and denote by $\mathrm{N} \theta$ the normal bundle of $\theta$ in $\mathbb{H}^{d}$. Each $\gamma^{t} \cdot H_{1}$ is a hyperbolic hyperplane containing $\theta$, and so it may be parametrized by its unit normal vector $v^{t} \in \mathbb{S}_{x}^{d-2} \subset \mathrm{N}_{x} \theta$ at $x$. The group $\Gamma$ acts isometrically on $\mathbb{S}_{x}^{d-2}$ : the image of $w \in \mathbb{S}^{d-2}$ is obtained by pushing back $\gamma^{t} \cdot w$, by parallel transport along $\theta$, from $\mathrm{T}_{\gamma^{t} \cdot x} \mathbb{H}^{d}$ to $\mathrm{T}_{x} \mathbb{H}^{d}$; so $v^{t}=\gamma^{t} \cdot v^{0}$. Examining the matricial form of such an isometry, one sees that

- either $\Gamma \cdot v^{0}=\left\{v^{t} ; t \in \mathbb{R}\right\}$ is periodic

- or it is not, and then its closure $\overline{\Gamma \cdot v^{0}}$, equal to its $\omega$-limit set $\bigcap_{n \in \mathbb{N}} \overline{\left\{v^{t} ; t \geqslant n\right\}}$, is diffeomorphic to a torus of dimension 2 or more.

In the first case, taking $t$ such that $v^{t}=v^{0}, \gamma^{t}$ is what we need: a loxodromic element stabilizing $H_{1}$. The second case is impossible for $\psi\left(\gamma^{t} \cdot H_{1}\right)=\gamma^{t} \cdot p_{1}$ would tend, when $t \rightarrow \infty$, to $q_{+}$: so $\psi$, which is continuous, would be constant, equal to $q$, on the $\omega$-limit set of $\Gamma \cdot v^{0}$, and hence equal to $q$ on $\overline{\Gamma \cdot v^{0}} \ni x$. This contradicts $\psi\left(H_{1}\right)=p_{1}$. This proves the claim.

By induction, we now finish the proof of proposition 5.1. As $G_{1}^{0}$ is a closed, connected and transitive subgroup of $\operatorname{Isom}\left(H_{1}\right)$, fixing no point at infinity on $\partial H_{1} \simeq \mathbb{S}^{d-2}$, by step 2 , it stabilizes a subsphere of $\mathbb{S}^{d-2}$ (and the hyperbolic subspace of $H_{1}$ that it defines) and acts transitively on this sphere. As $G_{1}^{0}$ acts transitively on $H_{1}$, this sphere is the whole $\partial H_{1} \simeq \mathbb{S}^{d-2}$. So we can apply lemma 5.3: $G_{1}^{0}$ acts transitively on $\mathrm{T}^{1} H_{1}$. In particular, the stabilizer $G_{v}$ of $v$ acts transitively on the unit vectors of $v^{\perp} \subset \mathrm{T}_{x} H_{1}$. Let $\left(v_{1}, \ldots, v_{d}\right)$ be a positive orthonormal basis of $\mathrm{T}_{x} \mathbb{H}^{d}$, with $v_{1}=v$. By induction, with $H_{k}=\exp \left(\operatorname{span}\left(v_{1}, \ldots, v_{k}\right)^{\perp}\right)$, it comes about that for each $k<d-1$, the stabilizer $G_{\left(v_{1}, \ldots, v_{k}\right)}^{0}$ of $\left(v_{1}, \ldots, v_{k}\right)$ acts transitively on the unit vectors of $\left(\operatorname{span}\left(v_{1}, \ldots, v_{k}\right)\right)^{\perp}$. Put together, steps $k=1$ to $k=d-2$ mean exactly that the stabilizer, $G_{x}$, of $x$ acts transitively on the direct orthonormal basis of $\mathrm{T}_{x} \mathbb{H}^{d}$. (Note that the induction stops with step $k=d-2$. Indeed, once the vectors $\left(v_{1}, \ldots, v_{d-1}\right)$ are fixed, $v_{d}$ is also as $G_{\left(v_{1}, \ldots, v_{d-1}\right)}^{0}=\{\mathrm{Id}\}$.) As $G$ acts transitively on $\mathbb{H}^{d}$ by lemma 5.3, $G$ acts then transitively on $\mathcal{B}^{+} \mathbb{H}^{d}$, which completes the proof. 


\section{Acknowledgments}

We thank one of the referees and Gregory McShane for their help.

\section{References}

[AVS] Alekseevskij D V, Vinberg E B and Solodovnikov A S 1993 Geometry of spaces of constant curvature Geometry II: Spaces of Constant Curvature vol 29, ed E B Vinberg and R V Gamkrelidze Encyclopaedia of Mathematical Sciences (Berlin: Springer) (Transl. from the Russian by V Minachin)

[BH] Benoist Y and de la Harpe P 2003 Adhérence de Zariski des groupes de Coxeter Preprint Université de Genève, www.unige.ch/math/biblio/?preprint/liste.html

[BGS] Ballmann W, Gromov M and Schroeder V 1985 Manifolds of nonpositive curvature Progress in Mathematics vol 61 (Boston, MA: Birkhauser)

[BBI] Bérard Bergery L and Ikemakhen A 1993 On the holonomy of Lorentzian manifolds Proc. Symp. on Pure Mathematics vol 54 (part 2) pp 27-39

[B1] Berger M 1955 Sur les groupes d'holonomie homogène des variétés à connexion affine et des variétés riemanniennes Bull. Soc. Math. France 83 279-330 (in French)

[B2] Berger M 1957 Les espaces symétriques non compacts Ann. Sci. Ecole Norm. Sup. 74 85-177

[DO] Di Scala A J and Olmos C 2001 The geometry of homogeneous submanifolds of hyperbolic space Math. Z. 237 199-209

[EN] Eberlein P and O’Neill B 1973 Visibility manifolds Pac. J. Math. 46 45-109

[G] Goldman W M 1999 Complex hyperbolic geometry Oxford Mathematical Monographs (New York: Oxford University Press, Oxford: Clarendon)

[GP] Gromov M and Pansu P 1991 Rigidity of lattices: an introduction Geometric Topology: Recent Developments (Montecatini Terme, 1990) (Lecture Notes in Mathematics vol 1504) (Berlin: Springer) pp 39-137

[K] Karpelevič F I 1953 Surfaces of transitivity of a semisimple subgroup of the group of motions of a symmetric space Dokl. Akad. Nauk SSSR (NS) 93 401-4 (in Russian)

[Ma] Matsumoto S 1992 Foundations of flat conformal structure Aspects of Low Dimensional Manifolds: Advanced Studies in Pure Mathematics vol 20, ed Y Matsumoto and S Morita, pp 167-261

[Mo] Mostow G D 1955 Some new decomposition theorems for semi-simple groups Mem. Am. Math. Soc. 14 $31-54$

[Z] Zeghib A Remarks on Lorentz symmetric spaces Compos. Math. at press

Zeghib A 2002 Preprint www.umpa.ens-lyon.fr/ ${ }^{\sim}$ zeghib/pubs.html 